\title{
APUNTES METODOLÓGICOS EN EL DESARROLLO DE EVALUACIONES DIFERENCIADAS
}

Notes on methodology development of differentiated evaluations

Notas sobre a metodologia de desenvolvimento de avaliaçôes diferenciada

\section{Rodrigo A. Espinoza Vásquez}

Universidad Central de Chile. Fono: +56 9 62410895. Correo electrónico:

respinoza.psicopedagogia@gmail.com

"Solo educadores autoritarios niegan la solidaridad entre el acto de educar y el acto de ser educados por los educandos"

Paulo Freire

\section{Resumen}

El desarrollo de la evaluación diferenciada en Chile es una herramienta que se ha asignado en el avance pedagógico de la enseñanza-aprendizaje toda vez que se detecta la presencia de alguna Necesidad Educativa Especial (NEE), generándose una barrera hacia el aprendizaje, ya que el estudiante no cuenta con las mismas oportunidades que el resto de sus compañeros, por lo tanto se deben realizar procedimientos evaluativos diversificados para que el aprendizaje escolarizado pueda ser considerado en su avance. Uno de los procedimientos propuestos corresponde a la metodología de Aprendizaje Basado en Equipos, pues integra elementos de la escuela tradicional formal con otros de tipo colaborativo, permitiendo que el estudiante vivencie la oportunidad de ser evaluado de una manera justa, considerando las NEE que pueden surgir en el proceso de enseñanza y aprendizaje.

Palabras clave: evaluación diferenciada, metodología, aprendizaje basado en equipos, mediación, discrepancia evaluativa.

\begin{abstract}
The development of differentiated evaluation in Chile is a tool that has been assigned to the educational advancement of teaching and learning since the presence of any Special Educational Need (SEN) is detected, generating a barrier to learning, as the student does
\end{abstract}


not have the same opportunities as the rest of their companions, therefore diversified evaluation procedures should be performed so that the learning can be considered enrolled in their advance. One of the proposed procedures corresponds to the methodology Team Based Learning, integrating elements of the traditional formal school with other collaborative type, allowing the student experience the opportunity to be assessed fairly, considering SEN that may arise in the process of teaching and learning.

Keywords: differentiated assessment, methodology, team based learning, mediation, evaluative learning discrepancy.

\section{Resumo}

O desenvolvimento da avaliação diferenciada no Chile é uma ferramenta que foi atribuído ao avanço educacional de ensino e aprendizagem desde a presença de qualquer Necessidade Educativas Especiais (NEE) é detectada, gerando uma barreira para a aprendizagem, como o estudante não tem as mesmas oportunidades que o resto de seus companheiros, pois diversificou procedimentos de avaliação deve ser realizada de modo que a aprendizagem pode ser considerado inscrito no seu avanço. Um procedimento corresponde à metodologia proposta aprendizagem baseadas nas equipes, integrando elementos da escola formal, tradicional, com outros tipos de colaboração, permitindo que o aluno vivencie a oportunidade de ser avaliado de forma justa, considerando NEE que possa surgir no processo de ensino e aprendizagem.

Palavras-chave: diferenciadas metodologia de avaliação, com base em equipamentos, a mediação, a discrepância de avaliação de aprendizagem.

\section{A modo de Introducción}

La Evaluación Diferenciada (ED en adelante) ha sido considerada un elemento asociado a las NEE, donde se requiere que pedagógicamente se minimicen las barreras en la evaluación, adaptándola a las necesidades que requieran los/as niños/as. Esto, se ha constituido en un elemento complejo en su aplicación en la educación formal chilena. Principalmente sus acciones no logran satisfacer las necesidades reales y se producen tensiones en el aprender, donde los principales perjudicados son aquellos niños que ven el aumento de barreras evaluativas en su recorrido escolar. 
Dicho trabajo se compone de un análisis situado en la realidad educativa de un tercer año básico, donde en términos cuantitativos existe la mayor cantidad de niños que cuentan con ED. El informe se sustenta bajo una metodología descriptivo-exploratoria, intencionando la participación de tres estamentos fundamentales, a saber: estudiantes, apoderados y profesores, éste último abordado desde la perspectiva de la Asignatura de Lenguaje y Comunicación, representado por el trabajo colaborativo con la profesora respectiva y la disposición de la Dirección para hacerlo realidad, donde el Departamento de Orientación actuó como eje articulador entre ellos.

Inicialmente se organiza un diagnóstico de la situación observada, analizando la perspectiva desde los tres actores antes mencionados, confluyendo ellos en las acciones y situaciones que ocurren dentro de la sala de clases.

Los elementos evidenciados mediante la recopilación de información (entrevistas, encuestas, observación participante, entre otros) permitieron configurar un escenario fértil de aprendizajes, los que en su generalidad veían la dificultad de replegarse sobre sí mismos y articular nuevos elementos de enseñanza, en el que niños con diagnóstico ${ }^{1}$ asociado a Necesidades Educativas Específicas: Déficit Atencional, Dificultades de Aprendizaje y Síndrome de Asperger, pudieran sacar fuera lo mejor de sí mismos en términos escolares.

Se organiza la información para dar pie a las acciones respectivas y que proponen en su esencia la democratización de los procesos evaluativos, debido a que se aprecia que el estilo de liderazgo predominante en la Dirección es jerárquico, dejando muchas veces las sugerencias de los profesores sin efecto. Se apuesta entonces, por un liderazgo distribuido que materialice las visiones de la gran mayoría de los implicados.

En términos concretos se hace uso de la metodología llamada Aprendizaje Basado en Equipos ( $\mathrm{ABE}$ en adelante) el que permite considerar elementos de la evaluación tradicional - hecho que fue comprobado como el de mayor valoración por apoderados y estudiantes- combinado con acciones de carácter activo y autoorganizativo en los

\footnotetext{
${ }^{1}$ Aun cuando el concepto de diagnóstico se refiere al "arte de conocer la naturaleza de una enfermedad mediante la observación de los síntomas y sus signos" (http://lema.rae.es/drae/?val=diagnóstico), la intención de utilizar esta palabra tiene que ver con las definiciones que han sido explicitadas en el decreto 170, lo que no necesariamente implica asumir el paradigma psiquiátrico que persiste en la escolarización.
} 
estudiantes, variando de manera significativa las disposiciones, dinámicas y resultados de calificación hacia mayores logros.

Se desarrolló como idea general la construcción estereotipada que ha ido cobrando la ED, pues existen consideraciones hacia ella que están valoradas en líneas de acción diferentes. Asimismo el foco de estudio está puesto en la tensión que se crea dentro de las salas de clases. Por una parte el sistema de evaluaciones constantes ha generado situaciones de estrés en los niños, pero más aún en aquellos que requieren de apoyos extra, derivados de las NEE presentes. El conflicto se genera por lo vertiginoso del modelo evaluativo del colegio, impidiendo que los estudiantes puedan retrotraer los aprendizajes logrados y evidenciados en las pruebas, ya que aquel que no logra el avance esperado empieza a ver limitadas sus posibilidades de mejora, ya que evaluativamente hay mínimas posibilidades que desde la evaluación diferenciada, le permitan volver sobre los temas ya vistos. Lo interesante es que este tema se comienza a aclarar progresivamente y de modo conversacional, mediante los talleres y las reuniones que se generan con los involucrados.

Estas acciones basan su desarrollo en la literatura y experiencia existente que entrega en el terreno educativo la gestión y el liderazgo. Herramientas que son operacionalizadas a partir del planteamiento de objetivos, acciones y procedimientos evaluativos diseñados exclusivamente para estos efectos.

\section{Contexto histórico de las NEE}

Un primer paso para entender la evaluación diferenciada es situarla en su dimensión histórica. Desde el Informe Warnock (1978) donde se asume que todo niño es educable hasta la aparición del Diseño Universal de Aprendizaje (DUA) como herramienta modificante de las disposiciones pedagógicas hacia la atención de la diversidad, han habido intentos por aplicar evaluaciones diferenciadas con mayor o menor éxito.

Las NEE entendidas como "todos aquellos apoyos que necesita un estudiante que se encuentra en una situación de desventaja para aprender, respecto de su grupo de referencia", contiene una raigambre solidaria. Es la expresión de la equidad de oportunidades para aprender de las que dispone un estudiante. Las NEE transitan en un terreno complejo. Por una parte, han sido erróneamente asumidas como características propias del estudiante, pero por otra -y en la línea que propone el Informe Warnock- como 
aquellas barreras que impiden el aprendizaje del estudiante, pudiendo ser éstas internas o externas. La visión sobre un estudiante que ve dificultoso su camino para aprender y que de una u otra forma se esfuerza por lograrlo convierte su trabajo en una opción dignificante de la educación formal.

\subsection{La dinámica de la evaluación}

La evaluación, parafraseando a Santos Guerra, asume un valor de uso y otro de cambio. El primero se orienta hacia la finalidad de juicio y toma de decisiones; el segundo, hacia el trueque. Se ha ido asociando la idea de equiparar los conceptos de evaluación y calificación. De hecho muchos profesores cotidianamente hablan de evaluar cuando se aplican pruebas y eso, el estudiante, lo ha captado en su esencia. Evaluar no es calificar, no obstante sabe un estudiante que al ser evaluado existirá la consecuencia de la nota, por ende, la situación de trocar la aplicación de un procedimiento o instrumento evaluativo en una referencia numérica se ha convertido en parte de la cotidianidad evaluativa. Es en este contexto que la evalaución diferenciada se gesta y surge como elemento funcional de la atención a las NEE con una carga diferenciada - como su nombre lo dice- cuando la situación particular de un estudiante lo exige. La necesidad de evaluar diferenciadamente asigna valores a los aprendizajes, toda vez que un estudiante que está presente en una sala de clases participa como tal y se le asignan roles que debe cumplir. Entre sus funciones asociadas se puede vislumbrar el sentido de trabajo, colaboración, disposición, atención, responsabilidad frente a la tarea, obediencia, entre otros. La escuela tradicional ${ }^{2}$ ha marcado la impronta de la conducta por sobre el aprendizaje, la inmovibilidad sobre la intranquilidad, considerando que desde el punto de vista arquitectónico y de la ergonomía, el educando está destinado al control corporal. Un niño que necesita evaluación diferenciada generalmente se mueve y desatiende porque tiene otras necesidades de aprendizaje. Por ende, la escuela se vuelve un obstáculo más que una ayuda, ya que en ocasiones el desconocimiento frente a las msituaciones de NEE es tal, que éstos ven cómo sus oportunidades de aprendizaje son vulneradas.

\footnotetext{
${ }^{2}$ Al usar el concepto "escuela tradicional" se hace referencia a los elementos y acciones que ocurren al interior de una sala de clases regular: estudiantes sentados uno detrás del otro, organización en el patio a modo de filas, formación antes de entrar a la sala, saludo de buenos días en coro, generación de dinámicas de clases dirigidas, sublimación del error por sobre el aprender, cuantificación de las conductas y aprendizajes, entre otros.
} 
Para ir comprendiendo esta idea es necesario ir a la raíz lexical de los conceptos. El vocablo evaluación ${ }^{3}$ se inscribe en la "asignación de un valor a algo", en este caso, al aprendizaje, pero desde fuera. Hay un otro que asigna ese valor y por lo tanto, otro quien asume dicho juicio. En cuanto a valoración ${ }^{4}$ contiene la raíz etimológica latina de valere, que signifca "ser fuerte" y que se relaciona con la idea "no tener defectos, proteger al más débil", que por lo tanto asume una condición hegemónica de que ese otro (en este caso el adulto, profesor) tiene sobre el educando. Ambas acepciones comparten la idea de que tanto el evaluar como el valorar es expositivo.

\subsection{La evaluación diferenciada: un acto de deferencia}

La evaluación diferenciada basa su esencia en un acto de solidaridad y justicia socioeducativa, pues se hace parte de los hándicaps que pueden surgir en el aprendizaje escolarizado. Estas barreras comúnmente han sido asociadas a los estudiantes, ya que deviene de un paradigma médico-hegemónico el asumir la dificultad para aprender como un trastorno psiquiátrico, entendiendo que desde la pedagogía los niños asisten a la escuela para aprender, no para enfermarse ${ }^{5}$. Asimismo, muchas veces surge la pregunta sobre el ritmo que lleva una clase: ¿el profesor (colegio) va muy apurado o el estudiante va muy lento? Esto parece llevar a la idea que mientras más "materia se pasa" más se aprende, aunque no por rendir mejor, se aprende más. La idea de ir rápido, de no devolverse sobre los temas no aprendidos, de estar constantemente siendo invadido por múltiples variables: contenidos, pruebas, conversaciones, discursos, etcétera, aumentan la sensación de que algo está mal hecho. Y afortunadamente no son los niños y niñas, sino que las diversas formas, disposiciones, intereses, paradigmas y todo aquello que esté instalado en el diseño de la educación formal actual, traspasándose hacia el esquema mental del profesor a partir de la dinámica que se vive en la escuela. En este contexto el estudiante no tiene la posibilidad de elegir a sus profesores; el profesor, tampoco elige a sus estudiantes. No obstante, éste último tiene la gran responsabilidad y deber de estimular a sus estudiantes hacia un aprender real, pues él sí eligió ser profesor.

\footnotetext{
${ }^{3}$ Maldonado, C. (Ed.) (1997) Clave. Diccionario de uso del español actual (2º ed). Madrid: SM. Pág. 780

${ }_{5}^{4}$ http://etimologias.dechile.net/?valor

${ }^{5}$ Interesante es la propuesta que realiza Calvo (2009) al indicar que existen situaciones generadas por parte del educador que terminan enfermando al educando.
} 


\subsection{Dejando el protagonismo y el ego de lado}

Al generarse una situación de evaluación es necesario mirarla desde diversas perspectivas. En primer lugar cuando se evalúa ¿qué se evalúa? Se entiende que la línea directa va orientada hacia el conocimiento que el estudiante tiene sobre lo que ha apendido. Lo que el profesor le ha enseñado. Sin embargo, lo que surge es subjetividad disfrazada de objetividad. Aquellos contenidos y tal vez aprendizajes que se han dado a conocer en la sala de clases deben ser reconocidos, comprendidos y aplicados mediante el instrumento que se ha disupuesto para ello. Puede ser mediante una prueba, una disertación, un informe u otro dispositivo que se elija, pero la elección no es al azar. La elección está hecha desde la subjetividad del profesor hacia los estudiantes, atendiendo a la experticia que éste tenga para hacer un instrumento que pueda considerar la mayoría de los elementos que fueron vistos en clases. Esta objetividad evaluativa no es tal. Se camufla entre escalas y fórmulas, además de sendos estudios sobre cómo evaluar, porque finalmente el profesor evaluará aquello que considere más importante dentro de lo que disponen los programas de estudio, atendiendo nuevamente, a los esquemas que estime convenientes a partir de su propia cognición. Siendo así la dinámica, es válido que aquel estudiante que requiera de apoyos tenga la posibilidad de moverse en un espacio que le permita validarse como un ser que aprende, pero que necesita de otros tiempos y espacios para confirmarlo.

La idea de evaluar se ha ido enraizando en nuestra cultura con la valoración inmediata a través de la calificación. Siendo ésta la expresión más elocuente de que alguien está aprendiendo. Santos (2001:110) llama a este fenómeno valor de cambio. Una costumbre de minimizar todo lo aprendido a un número, que ubica al educando en una escala de 1 a 7 en el caso chileno.

Los estudiantes comunmente comparan sus calificaciones con sus compeañeros de curso. Los padres también. Se genera una dinámica de competición permanente entre hermanos (si es que los hay), compañeros, padres o cualquier otro que sea conocido por la familia del estudiante. El acto de comparar encierra relaciones de poder y sumisión, al establecer una jerarquía en la que el niño con mayores barreras de aprendizaje generalmente sale perdiendo. 
Al asumir una postura pedagógica constructiva la minimización del ego del adulto es una necesidad si lo que se quiere lograr es que el estudiante aprenda de verdad y no simule que aprendió algo que olvidará en una semana. Esto implica entender al estudiante como activo en su aprendizaje. Es él quien indaga y pregunta. El adulto orienta. Dejar el ego de lado no significa que el profesor pierda su identidad, sino que ayude al niño a identificarse a través de su aprendizaje. En cada paso que da, un nuevo comienzo se gesta.

\section{Una experiencia de evaluación diferenciada}

Para vivenciar la evaluación diferenciada como hecho pedagógico se realizaron diversas acciones de modificación metodológica, que tomaron como eje fundamental la acción de mediar en los aprendizajes de los estudiantes. Así es como la experiencia-investigación se llevó a cabo en un colegio de dependencia particular-subvencionada en la comuna de La Serena, IV región de Coquimbo, Chile. El universo total del establecimiento es de 1056 estudiantes, donde se consideró un curso en particular: cuarto año básico, con una población de 38 estudiantes, siendo 8 de ellos la muestra seleccionada. A su vez, se generó un trabajo colaborativo con los profesores de asignatura, de manera que se estableciera un vínculo diferente con los niños que caen en el etiquetaje tradicional respecto de condiciones particulares en que se encuentran sobre las NEE.

La metodología usada en la investigación se basó en una combinación de elementos cuantitativos y cualitativos, cuyo fin (Baptista et al 2010:544) "no es reemplazar a la investigación cuantitativa ni a la cualitativa, sino utilizar las fortalezas de ambos tipos de indagación combinándolas y tratándolas de minimizar sus debilidades potenciales", integrando elementos del contexto que permitan una profundización mayor de la realidad a analizar.

La recopilación de información consideró los intereses y opiniones de estudiantes, profesores y apoderados, para los cuales se diseñaron diversos instrumentos de recogida de datos.

\subsection{Aplicación de entrevistas a estudiantes}


En el caso de los estudiantes se aplicaron entrevistas de tipo abierta, que integraban temáticas relevantes sobre los procesos evaluativos vividos en el colegio. Es así como frente a la pregunta:

¿Cómo sientes que te ha ido este año en el colegio?

Indican en su mayoría que el desempeño ha sido favorable e incluso uno de ellos lo describe como maravilloso a partir de sus experiencias escolares.

Sobre la pregunta: ¿Qué entiendes por evaluación diferenciada?

Las opiniones son heterogéneas. Existe poca claridad sobre el concepto y lo qué significa en la práctica. No obstante, quienes tienen un conocimiento algo más acabado, relacionan la situación de prueba y la realización de un isntrumento diferente al resto, asumiendolo desde el supuesto.

Respecto de la forma en que son evaluados, existe una alta conformidad. Valoran los procedimientos que se utilizan para que se evidencia que la evaluación sea diversificada.

Al consultarles ¿Cómo te gustaría ser evaluado? Orientan sus preferencias en el uso de pruebas (de selección múltiple y combinada con desarrollos breves), para luego continuar con el trabajo (generalmente en grupos)

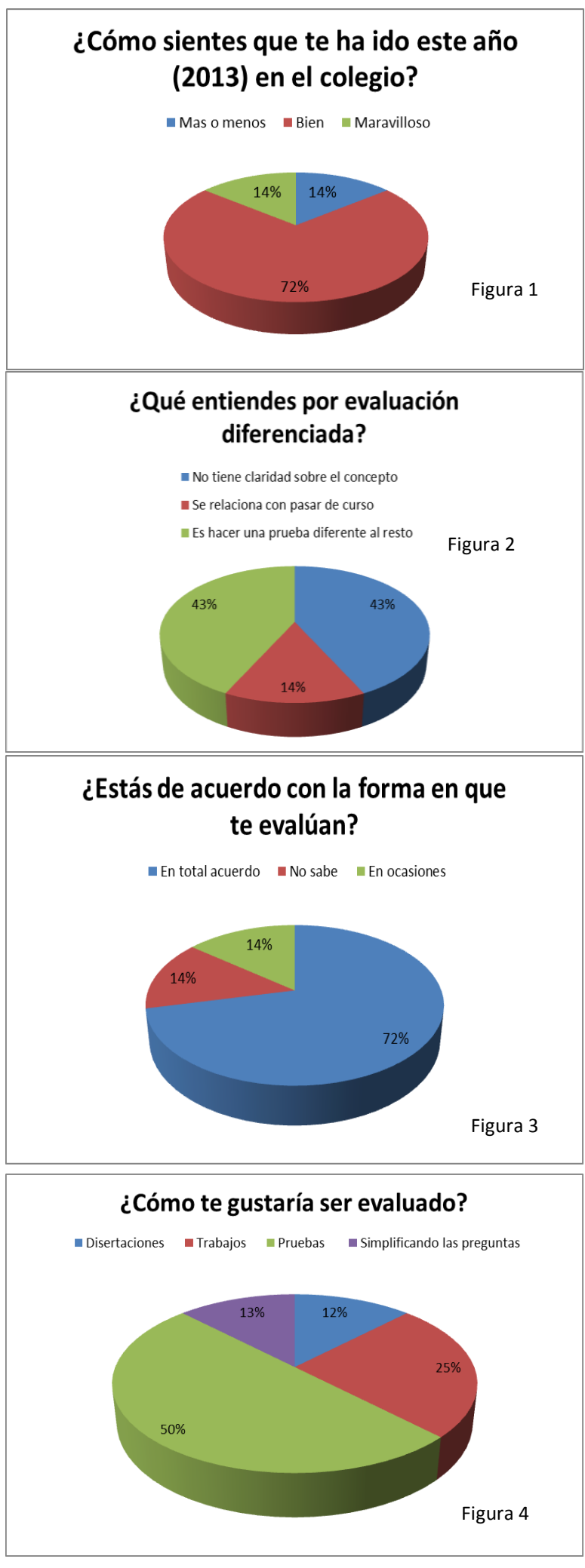


Finalmente se les consultó si ¿Crees que el trabajo en equipos mejora tus calificaciones

\section{y aprendizaje?}

A lo que respondieron de manera unánime que mediante el trabajo en equipos, podría ocurrir una mejora sustancial respecto de su aprendizaje, evidenciados a través de sus calificaciones

\section{A partir de la información entregada por} los estudiantes se desprenden las siguientes

¿Crees que el trabajo en equipos mejora tus calificaciones y aprendizaje?

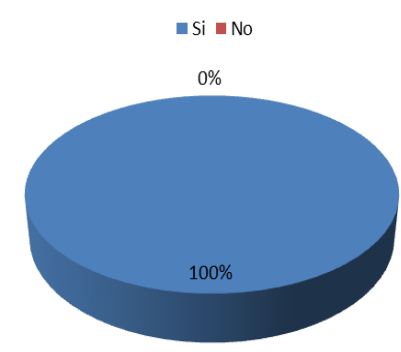

\section{ideas generales:}

- Existe una valoración positiva hacia el resultado del año académico, independiente de cómo fueron evaluados o cómo surgía la dinámica en sus clases.

- Entienden que la ED se traduce en algo concreto y que tiene que ver con que las pruebas varían, aumentando las posibilidades de mejora en cuanto a calificación

- Una gran mayoría está de acuerdo en la forma en que es evaluado. Es posible que esto suceda a partir de la baja diversificación que existe en evaluar, el acostumbramiento a un estilo característico, el desconocimiento por parte del niño y/o el profesor de otros procedimientos evaluativos. También se menciona en algunas entrevistas que el hecho de evaluar es visto como un acto de sinceridad por parte del profesor, donde sus mejores intenciones son que el niño aprenda y que éste, al estar en dicha situación pueda demostrarle al profesor que desarrollar una prueba es un voto de confianza depositado en sus estudios.

- Existe una alta valoración hacia la forma en que son evaluados, prefiriendo las pruebas a otros procedimientos. Se pide que se simplifiquen las preguntas y que los distractores sean mínimos, pues confunden. Punto coincidente con los apoderados (que se verá más adelante) que llama la atención por las reiteradas quejas hacia las pruebas de selección múltiple.

- Todos los entrevistados coinciden en que el trabajo en equipo mejora su aprendizaje y calificaciones, sintiéndose más seguros e integrados y con la posibilidad de ir solucionando sus dudas con sus compañeros o con el profesor como guía. 
Paralelamente se realizó un análisis cuantitativo de las calificaciones de la asignatura de Lenguaje y Comunicación en la que fue aplicada la metodología de Aprendizaje Basado en Equipos.

Durante el proceso de evaluaciones acumulativas del segundo semestre se realizó mediación durante las pruebas, no así en el primer semestre donde no se intervino al grupo de estudiantes. Se aprecia un aumento en la variación de las calificaciones de 0.4 décimas. Las razones pueden ser múltiples, entre ellas: dificultad en los contenidos, disposición hacia el aprendizaje, mediación inadecuada, entre otros.

\begin{tabular}{|c|c|c|c|c|c|c|c|c|c|c|c|c|c|c|c|}
\hline & \multicolumn{6}{|c|}{ Evaluaciones Acumulativas $^{6}$} & \multicolumn{6}{|c|}{ Evaluaciones Programadas } & \multicolumn{3}{|c|}{ Promedio } \\
\hline \multirow[t]{2}{*}{ Estudiantes } & \multicolumn{3}{|c|}{$1^{o}$ Semestre } & \multicolumn{3}{|c|}{$2^{o}$ Semestre } & \multicolumn{3}{|c|}{$1^{\circ}$ Semestre } & \multicolumn{3}{|c|}{$2^{\circ}$ Semestre } & \multirow[t]{2}{*}{$1^{o} S$} & \multirow[t]{2}{*}{$2^{o} S$} & \multirow[t]{2}{*}{ Var. } \\
\hline & $1^{o}$ & $2^{o}$ & Var. & $1^{o}$ & $2^{o}$ & Var. & $1^{o}$ & $2^{o}$ & Var. & $1^{o}$ & $2^{\infty 8}$ & Var. & & & \\
\hline $\mathrm{CA}$ & 5 & $J, 1$ & 0,1 & 5,1 & $\sqrt{3}$ & 0,3 & 4,3 &, 3 & 1 & 3,6 & 3,1 & $-0,5$ & 4,9 & 4,6 & $-0,3$ \\
\hline EA & $\overline{5,4}$ & 4,8 & $-0,6$ & 5,2 & 5,5 & 0,3 & 5,2 & 5,3 & 0,1 & 5,8 & 4,9 & $-0,9$ & 5,3 & 5,6 & 0,3 \\
\hline $\mathrm{CB}$ & 5 & 5,2 & $-0,2$ & 5 & 6,2 & 1,2 & 4,3 & 5,1 & 0,8 & 5,1 & 4,7 & $-0,4$ & 5 & 6,3 & 1,3 \\
\hline $\mathrm{VC}$ & 6,6 & 6,3 & $-0,3$ & 5,6 & 6,3 & 0,7 & 6,3 & 6,4 & 0,1 & 6 & 6,5 & 0,5 & 6,4 & 6,2 & $-0,2$ \\
\hline $\mathrm{IH}$ & 4,7 & 5 & 0,3 & 4,4 & 4,4 & 0 & 5,2 & 4,8 & $-0,4$ & 5,5 & 2,8 & $-2,7$ & 4,9 & 4,5 & $-0,4$ \\
\hline $\mathrm{VC}$ & 5,3 & 4,8 & $-0,5$ & 5,9 & 5,1 & $-0,8$ & 4,3 & 5,4 & 1,1 & 5,8 & 4,3 & $-1,5$ & 5,1 & 5,4 & 0,3 \\
\hline JT & 4,9 & 5,1 & 0,2 & 4,3 & 5,1 & 0,8 & 3,3 & 4,6 & 1,3 & 3,8 & 3,2 & $-0,6$ & 4,3 & 4,2 & 0,1 \\
\hline Promedio & 5,3 & 5,2 & $-0,1$ & 5,1 & 5,4 & 0,3 & 4,7 & 5,3 & 0,6 & 5,1 & 4,2 & $-0,9$ & 5,1 & 5,2 & 0,1 \\
\hline
\end{tabular}

En las evaluaciones programadas se realizó mediación en la primera evaluación del segundo semestre, no así en la segunda, pues se pretendió correlacionar las calificaciones y el impacto en la nota que pudiese tener la mediación, que en este caso muestra un descenso importante, incluso con lo sucedido en el primer semestre, generando una distancia de 0.9 décimas de nota en términos de promedio y acumulado por el grupo total de estudiantes. Aun cuando los contenidos no son comparables, sí lo es la situación de prueba, ya que los niños desarrollan este trabajo bajo el mismo formato y con preguntas semejantes (comprensión lectora a nivel explícito e implícito, uso de vocabulario y redacción modelada). A modo de idea general, se permite asociar a la mediación como un

\footnotetext{
${ }^{6}$ Aplicación de tres pruebas que luego son promediadas para obtener una nota

${ }^{7}$ Corresponde a una sola prueba que consolida los contenidos por unidad y equivale a una nota de pruebas evaluativas, es decir, tres pruebas acumulativas

${ }^{8}$ Evaluación realizada el 13 de diciembre de 2014, último día de clases
} 
articulador efectivo en la mejora de las calificaciones, situación que puede también ser ampliada hacia el aprendizaje escolarizado de la asignatura en cuestión.

\subsection{Aplicación de instrumentos de recogida de información a profesores}

En cuanto a la indagación con los profesores respecto del nivel de conocimiento y el desarrollo de mejoras para la aplicación de evaluaciones diferenciadas efectivas, se utilizó una encuesta. Además se entrevistó a una profesora como informante clave, donde se identifican los siguientes resultados:

Ideas generales a partir de lo investigado, donde los profesores expresan que:

- Conocen el concepto de evaluación diferenciada y las implicancias que tiene para su trabajo.

- Aplican ED en sus clases cuando el Departamento de Orientación ha informado al respecto, aunque la efectividad de este procedimiento es percibido como relativo por ellos.

- Utilizan la Pauta de Registro para ED, aunque consideran que las indicaciones no se ajustan a la realidad del aula a cabalidad.

- Consideran que cuentan con un bajo nivel de capacitación y especialización para realizar un proceso más efectivo, además de la posibilidad de conocer a cabalidad los diagnósticos de los estudiantes. Asumiendo mayor proactividad el Departamento de Orientación en este aspecto y desarrollando jornadas de capacitación.

- Proyectan la aplicación real y efectiva de la ED en un horizonte de dos años. Los seis primeros meses y el año posterior, no son suficientes y se apuesta a que en dos años debiese mejorar este aprendizaje a nivel organizacional.

- Aunque la mayoría no asume como trabajo extra la realización de ED, los profesores expresan que no cuentan con el tiempo y espacio suficiente para llevar adelante esta tarea. Esto genera una contradicción evidente, pues permite inferir que en rigor sí la consideran como trabajo extra. 
- Existe voluntad para generar un trabajo colaborativo con otros profesionales, además de la creación de un "banco de pruebas" o dispositivo ad hoc que permita simplificar el tiempo invertido en realizar nuevas evaluaciones.

El registro de ED mediante pautas se entiende como una tramitación administrativa, poco reveladora de lo que se hace en el aula, aun no contando con la capacitación adecuada, pero conociendo lo que significa la ED. Esta situación es contradictoria, ya que se sigue un proceso sin tener la certeza de que esté en la línea adecuada. Los profesores necesitan simplificar este procedimiento, pudiendo generar otros instrumentos de manera rápida y en tiempo real, ojalá inmediata.

La disposición de tiempos y espacios es inexistente, a no ser que destinen el suyo propio para estos propósitos, donde además no hay una remuneración acorde que permita reorientar los recursos hacia esta acción.

Se declara disposición y necesidad de ser capacitado en ED, aunque de todas formas enfocan este cambio en el largo plazo.

\subsubsection{Ideas generales a partir de entrevista a informante clave: Profesora de} Educación General Básica (Asignatura de Lenguaje y Comunicación)

- Se asume que al ser diferenciada la evaluación y adaptada a las necesidades del estudiante, no es necesaria la mediación. En este sentido, un estudio sobre la Experiencia de Aprendizaje Mediado indica "que el sujeto no es estático o inmutable, razón por la cual, el ser mediado siempre será necesario” (Orrú, 2003:4)

- La aplicación de ED se basa en la asignación de mayor tiempo. Rara vez existe la creación de un nuevo instrumento.

- La coherencia entre prueba y diagnóstico de NEE se basa en la supervisión, no generándose instrumentos ad hoc a las necesidades del niño.

- Las estrategias de ED corresponden a devolver la prueba o disponer espacios informales fuera del horario de clases.

- Asume la ED bajo un paradigma funcional: cuando los padres no están de acuerdo con las notas de sus hijos. 
- Las políticas que propone el colegio son consideradas como insuficientes, debiendo haber una persona destinada exclusivamente a este trabajo, donde además no existe el tiempo necesario para hacerlo.

- La mediación es entendida como una alternativa, pero se desconfía de ella al ser una posibilidad de que el estudiante abuse en términos de que se le dé explícitamente la respuesta.

- Gran parte de las dificultades en ED se solucionarían si el Departamento de Orientación designara a un funcionario encargado formalmente de esto. Además se debe socializar por curso la información de los estudiantes con NEE.

- La disposición de los profesores hacia la ED es dispar, existiendo prejuicios. Unos lo hacen con mayor dedicación y otros, sólo por cumplir.

Se desprende de las ideas expuestas que la ED es entendida como un constructo que no está bien elaborado en el colegio. Existe una construcción estereotipada sobre el tema, suponiendo por ejemplo que el sólo hecho de asignar mayor tiempo es sinónimo de ED. También se aprecia el fenómeno de atribucionalidad externa, donde las responsabilidades no son de quien realiza las clases y las actividades, sino que de otro profesional que vendría a solucionar los problemas que no solo correspondería en acompañar al profesor en el diseño y propuesta evaluativa, ya que también se debe mediar con el estudiante. Situación de la cual se desconfía al ser entendida como una actividad que regala las respuestas a las preguntas, cuando esto corresponde a la simplificación organizacional y cognoscitiva respecto de cómo un estudiante capta la información de diversas maneras.

Existen prejuicios asociados al concepto de ED, entre ellos: la disposición de diagnósticos hechos a la medida por profesionales externos que son cuestionados por los profesores, el uso de la calificación como significante de logro por parte de los padres y competencia por parte de los educandos, la asignación de estrategias limitadas para que el niño rinda una mejor prueba, etcétera. A todo esto se suma que el Colegio exige el cumplimiento administrativo de este procedimiento, pero no se cerciora de que se haga efectivamente. Tal vez por la baja significancia que tiene, el exceso de trabajo para los profesores quienes además no son remunerados por esto, el bajo porcentaje de representatividad dentro del total de la matrícula $(7,1 \%)$, la falta de personal con dedicación 
exclusiva, la destinación de recursos extra para una acción que tiene una baja representatividad bajo los parámetros del SNED, el discurso academicista del establecimiento, el desconocimiento frente al tema de inclusión escolar, etcétera. Esto impacta directamente en la calidad evaluativa del establecimiento, donde además la gran cantidad de estudiantes limita la personalización de la enseñanza, quedando sólo en la recopilación de evidencia para efectos de supervisión estatal.

Al respecto, Senge (1992:10) indica que las organizaciones adolecen de problemas de aprendizaje, donde sus principales causas son:

- La concentración específica en sólo un puesto de trabajo, disminuyendo la posibilidad de pensamiento sistémico.

- "El enemigo externo": donde se culpa de los fracasos al resto por la propia inoperancia.

- La ilusión de hacerse cargo, en cuanto a ser proactivo, pero que en el fondo no es más que la reactividad frente a los problemas y que pocas veces impiden mirarse en términos personales.

- La fijación en los hechos, situación en la cual se asume que detrás de cada hecho hay una causa obvia, con la consecuente sensación de inmediatez en la solución de los problemas.

- La parábola de la rana hervida. Reaccionando de manera explosiva frente a los cambios radicales, no así cuando estos se van haciendo progresivamente.

- La ilusión de que "se aprende con la experiencia". La experiencia sin duda es un potente medio de aprendizaje, pero cuando ya no vemos las consecuencias de nuestros actos trascendiendo el horizonte de nuestros aprendizajes, se dificulta el volver a aprender de la experiencia directa que se basa en la confusión y el error.

- El mito del equipo administrativo. Bajo el concepto de incompetencia calificada (Argyris) se conforman equipos que solucionarán los problemas, pero que bajo la complejidad prefieren callar y ceder, pues es mejor simular que se sabe a que se ignora.

En el caso evidenciado de los profesores y según lo que plantea el autor, hacer una revisión organizacional en torno a la ED implica que, a modo de diagnóstico, como 
organización nos enfrentamos a un problema de aprendizaje, ayudado por la escasez de espacios de conversación y demostrado por las siete categorías que caracterizan esta situación, las que se deben tener en cuenta para que el proceso de posterior mantención de las acciones propuestas no se vean solo como intenciones y terminen siendo parte de una historia con final abrupto.

\subsection{Aplicación de instrumentos de recopilación de información para padres y} apoderados

Uno de los actores principales corresponde a los apoderados de los estudiantes. Muchos de ellos hacen grandes esfuerzos para que la asistencia de sus hijos al colegio sea provechosa y de calidad, por ello se considera que la consulta sobre la percepción de este proceso, es vital. Es así como a través de una encuesta se obtiene la siguiente información:

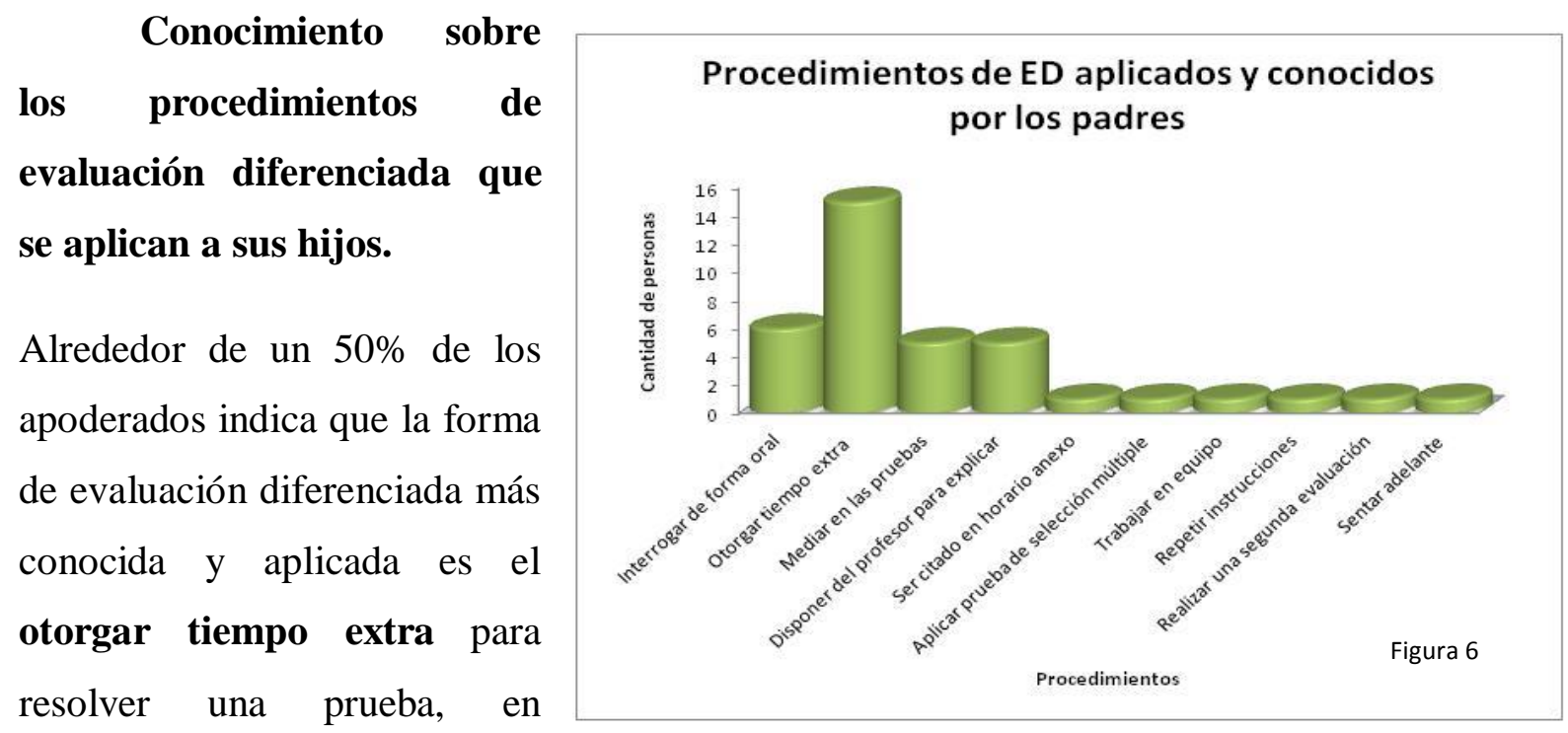

segundo

lugar con $15 \%$ lo sigue la interrogación oral y a continuación la mediación en las pruebas. 


\section{Satisfacción sobre los procedimientos de evaluación y calificación:}

\begin{tabular}{|c|c|c|c|c|}
\hline $\begin{array}{c}\text { Instrumento de } \\
\text { evaluación }\end{array}$ & Promedio $^{9}$ & $\begin{array}{c}\text { Cuantificación en } \\
\text { rango de } \\
\text { valoración }\end{array}$ & $\begin{array}{c}\text { Rango de } \\
\text { valoración }\end{array}$ & $\begin{array}{c}\text { Ordenamiento } \\
\text { por } \\
\text { preferencia }^{11}\end{array}$ \\
\hline Otro & & S/R & S/R \\
\hline $\begin{array}{c}\text { Prueba de } \\
\text { selección múltiple }\end{array}$ & 3,3 & 2 & Media & 1 \\
\hline Disertación & 3,4 & 2 & Media & 2 \\
\hline Trabajo en equipo & 4,1 & 2 & Media & 3 \\
\hline Proyecto & 4,5 & 2 & Media & 4 \\
\hline Pruebas mixtas & 4,6 & 2 & Media & 5 \\
\hline Investigación & 4,9 & 2 & Media & 6 \\
\hline Interrogación oral & 5 & 2 & Media & 7 \\
\hline Prueba de & 5,5 & 2 & Media & 8 \\
\hline desarrollo & & 2 & Media & 9 \\
\hline Informe escrito & 5,8 & & & \\
\hline
\end{tabular}

Los apoderados consultados le asignan una preferencia alta a las pruebas de selección múltiple, aun cuando en las reuniones las críticas están centradas en este tipo de evaluación por la forma en que se plantean las preguntas, ya que se utiliza un lenguaje complejo que confunde al estudiante; aun así, se sigue prefiriendo este formato. Otro punto que llama la atención es el lugar que ocupa la interrogación oral, pues siendo una de las más utilizadas no genera satisfacción entre los apoderados. Esto tal vez se deba a que al ser interrogado el estudiante los criterios de evaluación se vuelvan altamente subjetivos, porque es percibido como "un favor" en el caso de algunos profesores, por lo que la garantía de objetividad relativa es mínima.

\subsubsection{Categorías de análisis e interpretación de lo expuesto por los apoderados}

\footnotetext{
${ }^{9}$ Comprendido entre 1 y 8 , donde las representaciones de mayor satisfacción sobre los instrumentos de evaluación comienzan en 1

${ }^{10}$ Categorizado bajo el procedimiento de escala Lickert, donde 2 representa una valoración media

${ }^{11}$ Ordenamiento en base a las preferencias. El orden es correlativo y ascendente.

${ }^{12}$ Los apoderados no indican otros medios de evaluación distintos a los propuestos.
} 
Las categorías aquí propuestas son originales y se concibieron como ideas generales que organizan las temáticas expresadas por los apoderados mediante los instrumentos de recogida de datos que se utilizaron. Se presenta la categoría y su respectivo análisis, de manera que permita la comprensión de las ideas que ellos proponen.

\begin{tabular}{|c|c|c|c|c|c|}
\hline $\begin{array}{c}\text { Categorías } \\
\text { de } \\
\text { análisis }\end{array}$ & $\begin{array}{l}\text { Normar el } \\
\text { procedimiento de ED }\end{array}$ & $\begin{array}{l}\text { Innovación } \\
\text { metodológica }\end{array}$ & $\begin{array}{l}\text { Evaluación } \\
\text { diversificada }\end{array}$ & $\begin{array}{l}\text { Comunicación } \\
\text { efectiva con } \\
\text { apoderados }\end{array}$ & $\begin{array}{l}\text { Aumento de } \\
\text { recursos }\end{array}$ \\
\hline & $\begin{array}{l}\text { Se sugiere un mayor } \\
\text { conocimiento de las } \\
\text { situaciones de los } \\
\text { estudiantes, de } \\
\text { manera que el trabajo } \\
\text { hecho por el profesor } \\
\text { sea orientado en base } \\
\text { a ese conocimiento, } \\
\text { el que además debe } \\
\text { ser normado para no } \\
\text { depender } \\
\text { exclusivamente de la } \\
\text { voluntad de cada } \\
\text { profesor, sino que de } \\
\text { un criterio } \\
\text { consensuado y } \\
\text { conocido por todos. }\end{array}$ & $\begin{array}{l}\text { El uso de } \\
\text { acciones } \\
\text { metodológicas } \\
\text { diversas, que } \\
\text { permitan el } \\
\text { accionar } \\
\text { cognitivo, motriz } \\
\text { y emocional del } \\
\text { niño son } \\
\text { necesarios para } \\
\text { que } \\
\text { consecuentemente } \\
\text { varíe la forma en } \\
\text { que se evalúa. } \\
\text { Apuntar hacia el } \\
\text { desarrollo de } \\
\text { destrezas y } \\
\text { habilidades, } \\
\text { permite ampliar el } \\
\text { camino basado en } \\
\text { el contenidismo. }\end{array}$ & $\begin{array}{l}\text { Buscar la } \\
\text { participación } \\
\text { del } \\
\text { estudiante, } \\
\text { ser coherente } \\
\text { con el } \\
\text { diagnóstico } \\
\text { en la } \\
\text { evaluación, } \\
\text { simplificar el } \\
\text { lenguaje y las } \\
\text { formas. En el } \\
\text { fondo mayor } \\
\text { participación } \\
\text { con menores } \\
\text { trabas es lo } \\
\text { que se pide } \\
\text { para los } \\
\text { estudiantes } \\
\text { que cuentan } \\
\text { con ED. }\end{array}$ & $\begin{array}{l}\text { Ser parte activa, } \\
\text { al igual que los } \\
\text { educandos es lo } \\
\text { que necesitan los } \\
\text { apoderados. } \\
\text { Estar al día en } \\
\text { técnicas para } \\
\text { ayudar a sus } \\
\text { hijos y atentos a } \\
\text { la información, } \\
\text { se asume como } \\
\text { un pilar } \\
\text { fundamental para } \\
\text { que el niño } \\
\text { pueda mejorar } \\
\text { sus aprendizajes. } \\
\text { Existe } \\
\text { propensión a } \\
\text { cooperar. }\end{array}$ & $\begin{array}{l}\text { Un mayor y } \\
\text { mejor } \\
\text { cobertura se } \\
\text { asume desde } \\
\text { la } \\
\text { contratación } \\
\text { de nuevos } \\
\text { profesionale } \\
\text { s que } \\
\text { puedan } \\
\text { atender las } \\
\text { necesidades } \\
\text { de los } \\
\text { estudiantes. }\end{array}$ \\
\hline
\end{tabular}

\section{El Aprendizaje Basado en Equipos como opción de evaluación diferenciada}

Una de las posibilidades que acerca la idea de evaluación a la empatía y la justicia pedagógica, se relaciona con las estrategias metodológicas que se pueden implementar en 
clases. En este caso se puede hablar del Aprendizaje Basado en Equipos ${ }^{13}$ cuya propuesta toma elementos de la escuela tradicional y los combina con otros de carácter colaborativo. Integra la realización de pruebas de alternativas de forma individual, la que luego es desarrollada en grupos organizados por características de estilos de aprendizaje, donde los estudiantes trabajan en equipo al resolver las problemáticas que se proponen. Posteriormente, se realiza una revisión grupal de las preguntas y respuestas que se incluyeron en la prueba y se realiza la retroalimentación, donde el estudiante dispone de un espacio de apelación sobre aquellas discrepancias que ocurran en la dinámica de la retroalimentación, para finalizar con una miniclase, profundizando aquellos aspectos que quedaron poco claros o donde los estudiantes tuvieron mayores consultas. En términos cuantitativos se promedian las calificaciones de ambas pruebas, asignándole un $50 \%$ cada una. Cualitativamente se destaca la participación de los estudiantes, las ganas de compartir y colaborar con sus compañeros, ayudando a quien dispone de tiempos menos acelerados para tal o cual aprendizaje escolar.

\subsection{Un acercamiento metodológico}

Para hacer efectiva esta metodología es necesario considerar algunas modificaciones en los roles. Profesores y estudiantes conviven en una dinámica diferente.

El rol primario del profesor cambia desde la provisión de la información hacia el diseño y gestión del proceso instruccional en general, y los roles de los estudiantes cambian desde recipientes pasivos de la información a responsables de estudiar los contenidos del curso en forma autónoma de tal forma que puedan estar preparados para el trabajo grupal en clases. No obstante estos cambios no son automáticos, ya que implica que al menos cuatro componentes fundamentales se puedan implementar de manera ideal en este procedimiento (Michaelsen y Sweet, 2012:2)

Los elementos a considerar son:

- Grupos: Los grupos deben ser formados y guiados en forma apropiada.

\footnotetext{
${ }^{13}$ Se realizaron adaptaciones del modelo original propuesto por Michaelsen y Sweet en estudiantes de Educación Básica, considerando que este modelo ha sido trabajado en su gran mayoría en Educación Superior.
} 
- Responsabilidad: Los estudiantes deben ser responsables por la calidad de su trabajo individual y grupal.

- Retroalimentación: Los estudiantes deben recibir retroalimentación frecuente y oportuna.

- Diseño de tareas y actividades: Las tareas y actividades grupales deben promover tanto el aprendizaje y el desarrollo del equipo.

Estas acciones impactan en la adherencia que puedan presentar los estudiantes en las actividades de clases, privilegiando el trabajo colaborativo e indagatorio; potenciando un aprendizaje activo por parte de los estudiantes. Tal vez el elemento diferenciador con otras metodologías sea la responsabilidad que se le asigna al estudiante, puesto que debe realizar estudios previos a las evaluaciones, pero que tendrán una incidencia directa en la sinergia que se genere al interior del equipo, ya que privilegia el aprendizaje entre iguales, siendo el profesor un mediador de las conversaciones que surjan entre los estudiantes.

La dinámica de clases bajo el enfoque que propone el Aprendizaje Basado en Equipos (ABE) implica que los grupos no sean formados de manera azarosa, sino que a través de criterios claros. En el caso de la experiencia presentada, se realizaron bajo la identificación de estilos de aprendizaje. El modelo utilizado fue el de Kolb (Castro y Guzmán, 2005:88), caracterizado por los estilos:

- Acomodador-Dinámico

- Convergente-Activo

- Asimilador-Analítico

- Divergente-Reflexivo

En el caso de los dos primeros se hace referencia a la experimentación activa, que puede avanzar desde elementos concretos a otros más abstractos. Para los dos restantes, una característica común es la observación reflexiva, moviéndose desde la conceptualización abstracta hacia la experiencia concreta, según la forma en que están presentados anteriormente. De esta manera el aprendizaje se vivencia de una manera diferente según 
cada persona. En el caso puntual que se expone cada equipo incluyó al menos un niño por cada estilo, repitiéndose en algunos de ellos por la cantidad de estudiantes en el curso.

Es así, como se siguieron de manera secuenciada los pasos que propone el $\mathrm{ABE}$, implementando las fases propuestas en el Proceso de Aprendizaje Inicial (Michaelsen y Sweet, 2012:5)

- Lecturas requeridas: si bien se propone una lectura previa de los temas a tratar, se optó por utilizar las clases previas a las evaluaciones realizadas, de manera que los problemas planteados se fueran resolviendo clase a clase.

- Examen individual: se consideraron pruebas de selección múltiple para estos efectos, ya que permite combinar diversos niveles de dificultad, además de ser la confluencia que se registró en la recogida de información con los tres actores involucrados: familia, profesores y estudiantes. Estos últimos rinden un examen individual que permite tener una referencia sobre el conocimiento que disponen en términos conceptuales.

- Examen grupal: los educandos rinden el mismo examen, pero grupalmente. El trabajo colaborativo y autoorganizacional es el objetivo a cumplir. Es el punto de quiebre donde se visualiza plenamente el trabajo en equipo. Luego se hace una revisión grupal, que en este caso debió ser adaptada con el uso de pizarras, ya que el diseño original propone el uso de cartillas con las respuestas ya dadas, pero ocultas con una cobertura semejante a la que tienen los raspes de los juegos de azar. Esta adaptación permite generar una diferencia en el cómo acceden los educandos a una respuesta, valorando la confianza como elemento esencial en la dinámica de revisión de prueba.

- Proceso de apelación: en este punto los estudiantes tienen acceso a sus materiales, ya sean cuadernos, libros u otro material didáctico para corroborar sus respuestas, pudiendo discutir con el profesor aquellas respuestas que generen dudas.

- Retroalimentación del profesor: la última fase de este proceso es donde el profesor genera un diálogo con los estudiantes y retroalimenta todas aquellas dudas que surgen de la revisión previa. También se puede optar por una miniclase, 
poniendo en relevancia aquellos temas más importantes que son necesarios de profundizar.

\subsection{La retroalimentación basada en la confianza}

La generación de este procedimiento permite en gran medida disminuir la carga laboral del docente. Tal como indican investigaciones al respecto, donde

Los docentes reconocen la importancia de la evaluación diferenciada, pero acusan básicamente a la falta de tiempo, de no poder planificar y elaborar procedimientos que permitan facilitar el acceso a una evaluación pedagógicamente justa y que dé respuesta a la diversidad, así como también, a la falta de conocimiento sobre el tema y de estrategias relacionadas en este ámbito (Calderón, 2011:34)

La elaboración de tales dispositivos evaluativos consiste en realizar labores tradicionalmente asumidas en el espacio extraescuela, restándoles tiempo y espacio de vida personal a los profesores. Por ello, esta metodología propone que la realización de la ejecución de la prueba, la revisión y la retroalimentación se haga en el mismo momento y lugar que se realizan las diversas tareas de evaluación. Se asocia además, que un elemento valórico fundamental que debe primar es la confianza. Tanto el estudiante como el profesor organizan sus disposiciones en función de construir un espacio donde el engaño sea superado por la necesidad real de aprender, existiendo variadas oportunidades donde podrán conducirse las mejores intenciones de quienes aprenden y enseñan formalmente.

En el terreno de la sala de clases se evidencian los gestos y actitudes de los niños cuando necesitan ayuda y un compañero los acoge. Solo el acto de mirar y entender lo que se requiere es un universo en sí mismo. Cada representación coordinada de acciones integra espacios y tiempos de un lenguaje simple y complejo. Lo sutil va tomando forma y se convierte en aprendizaje. Ese que se muestra a través de los ojos, cuando se expanden y penetran en el lugar de ese otro que interpreta las acciones como una forma de relación que va más allá de una calificación.

La educación formal invisibiliza al estudiante, lo uniforma. La evaluación también hace lo propio, pero es en el conocimiento del profesor, que va derribando barreras, que 
hace justicia en el acto de aprender y/o enseñar, donde se va generando el avance de un aprendizaje más sincero. La propensión que cada persona tiene sobre esta última idea, es la puerta de entrada a un universo múltiple, que consolida al ser humano como una especie en un constante ir y venir de su historia. Asimismo, la experiencia vivenciada en la sala de clases en cuestión, invita a generar procesos inclusivos de una pedagogía solidaria, considerando por una parte que la acción de instalar en el escenario pedagógico el trabajo colaborativo y la mediación por parte de sus propios compañeros ayudan a entablar un dialógo fluido entre los diversos actores: estudiantes, profesores y padres.

Sobre estos últimos, se elicitan diversas ideas. Por una parte prefieren que sus hijos sean evaluados mediante pruebas de selección múltiple, pero con mediación de un adulto. El trabajo colaborativo está en segundo lugar de preferencias. Los padres se interesan en que sus hijos puedan ser evaluados diferenciadamente, realizando trabajos en conjunto con otros compañeros, pero con las reglas claras y orientados en todo momento. De hecho, al mantenerse la preferencia de pruebas de selección múltiple, se genera un hecho que llama la atención, a saber: la mantención de dispositivos evaluativos tradicionales y estructurados, frente a otros de mayor diversidad y en donde un estudiante puede explayarse sobre la respuesta en ciertos temas. Esto genera una contradicción, que he optado por llamar discrepancia evaluativa. Que corresponde a la situación donde los adultos (padres y madres en este caso) exigen que sus hijos sean evaluados de forma diferenciada y diversificada, pero prefieren que se haga con un instrumento estructurado y "objetivamente" confeccionado, pues al momento de reclamar una calificación, los criterios de argumentación se entienden como más certeros pues la respuesta subyace en la pregunta. Se infiere además, que la preocupación de los adultos se instala con mayor interés en la calificación que en el aprendizaje, ya que muchos coinciden que al momento de exigir alguna modificación ésta sea representada a través de una nota. Lo que no necesariamente expresa la calidad de los aprendizajes logrados en la escuela.

\section{Ideas generales a modo de conclusión}

La evaluación no está condicionada ni limitada a la entrega de un producto. No obstante, la realidad de nuestro país enfatiza en el resultado y la cuantificación de los aprendizajes para emitir juicios. Nada más alejado de lo que significa educación. Todos aquellos aprendizajes 
que se someten a la minimización del número son la evidencia de que alguien aprendió, pero quedan fuera de ella todas las observaciones, conversaciones y aprendizajes "secundarios" que un niño vivencia. Se habla de constructivismo cuando se vive aun en el empirismo. Es más, actualmente existe una obsesión por querer registrarlo todo. Debe evidenciarse la educación escolarizada para demostrar que la gente aprende. Es lo mismo que ocurre con esos niños cuyos padres consiguen los cuadernos del compañero más aventajado, pudiendo copiar los contenidos para tener el cuaderno al día, ¿es eso acaso, la demostración de que aprende?, ¿realmente aprendió en clases? Por ende, la evaluación es parte innegable del proceso de enseñar y aprender, pero el arraigo a las tradiciones son tan fuertes que la evaluación sigue siendo el momento de castigo, hegemonización y subyugación que propone la escuela actual, que despegarse de este molde se vuelve complejo. Aún así, en este contexto y debiendo hacer uso de una evaluación de tipo diferenciada, que a todo esto, es una especie de oasis pedagógico, ya que permite empatizar con quien es evaluado y adaptar condiciones a las necesidades reales de los estudiantes, se puede optar por una evaluación permanente y constante, entendiendo que ésta es un proceso que acompaña en todo momento el aprender.

Una de las opciones a este respecto, convive en la metodología de Aprendizaje Basado en Equipos, que adaptada a la educación básica toma elementos de evaluación formal tradicional y los integra al trabajo colaborativo, generando un ambiente de mediación y conversación que permite que el estudiante pueda desenvolverse de una manera menos restrictiva y por ende, aclarar aquellas dudas que surgen en el aprender.

La visión de los profesores y de los/as padres/madres se incluyen en esta dinámica, teniendo ambos la posibilidad de moverse en un terreno que ha signficado una trinchera permanente y hostil, ya que las conversaciones en este sentido no siempre han sido las mejores. Por una parte, los profesores pueden minimizar el trabajo evitando el tener que llevarse las pruebas a sus casas y revisarlas ahí, y; los/as padres/madres, haciendo eco de su discrepancia evaluativa, pueden conciliar las aprehensiones surgidas de la "subjetividad" en que se desarrolla la evaluación diferenciada.

Esta experiencia ha sido entendida en el marco de equiparar las posibilidades evaluativas de aquellos estudiantes que presentan dificultades en su aprendizaje escolar y 
que de una u otra forma, ven vulnerado el acceso a una educación de calidad en un contexto homogeneizante.

\section{Referecias bibliográficas}

Calderón, K. (2011) Evaluación diferenciada: discursos y prácticas de los docentes de enseñanza básica, en tres establecimientos educacionales de la corporación municipal de La Florida, en Revista Electrónica Diálogos Educativos, 22, s/p.

Calvo, C. (2009) Los procesos educativos y la emergencia de complejidades caóticas y autoorganizadas. Acción pedagógica, 18, s/p.

Calvo, C. (2012) Del mapa escolar al territorio educativo. Disoñando la escuela desde la educación. $4^{\mathrm{a}}$ edición La Serena, Chile: Universidad de La Serena.

Castro, S. y Guzmán, B. (2005) Los estilos de aprendizaje en la enseñanza y el aprendizaje: Una propuesta para su implementación. Revista de Investigación, 58, s/p.

Espinoza, R. (2014) Evaluación diferenciada: un acercamiento a las tensiones pedagógicas en base a una propuesta de cambio planificado. Tesis para optar al grado de Magister en Gestión y Liderazgo Educacional. Universidad Central de Chile, La Serena. Chile.

Baptista, P., Fernández, C. y Hernández, R. (2010) Metodología de la investigación, 50 edición. México: Mc Graw Hill.

Maldonado, C. (Ed.) (1997) Clave. Diccionario de uso del español actual $2^{\circ}$ edición. Madrid: SM

Michaelsen, L. K., \& Sweet, M. (2012). Elementos Esenciales del aprendizaje basado en equipos. Team-Based Learning Collaborative [Internet]. Huntington: Team-Based Learning Collaborative.

Orrú, S.E. (2003) Reuven Feuerstein y la teoría de la modificabilidad cognitiva estructural. Revista de Educación, 332, 33-54.

Ortiz, J. A. M., González, A. G., Marcos, A. P., Victoria, M., \& Nardiz, A. (2003). Aprendizaje basado en problemas: una alternativa al método tradicional. Revista de Docencia Universitaria, 3, 2. 
Santos, M.A. (2001) La escuela que aprende. $2^{\circ}$ edición. España: Morata

Senge, P. (1992) La quinta disciplina. Argentina: Gránica 